\title{
Distribution Equilibria of Metal Chelates with Thiazolylazo Dyes between Two Phases Formed from an Aqueous Micellar Solution of a Nonionic Surfactant
}

\author{
Tohru Saitoh*, Yoshiya Kimura*, Tamio Kamidate*, Hiroto Watanabe* and Kensaku HaRaguchi** \\ *Faculty of Engineering, Hokkaido University, Sapporo 060, Japan \\ **The Government Industrial Development Laboratory, Hokkaido, . Toyohira, Sapporo 004, Japan
}

\begin{abstract}
A dilute aqueous micellar solution of poly(oxyethylene) $=4$-nonylphenyl=ether with oxyethylene units of 7.5 (average, PONPE-7.5) was separated into two phases (aqueous and surfactant) at room temperature. The partition constants of thiazolylazo dyes and their metal chelates between the two phases were determined at $293 \mathrm{~K}$ and an ionic strength of $0.1\left(\mathrm{NaClO}_{4}\right)$. Though a linear relationship was obtained between the partition constants of the chelating reagents in the extraction with PONPE-7.5 and those in the ordinary extraction with octanol, such a relationship was not obtained for the chelates. This suggested that the properties of the PONPE-7.5 phase as an extracting solvent were different from those of ordinary organic solvents.
\end{abstract}

Keywords Distribution equilibrium, poly(oxyethylene)=4-nonylphenyl=ether, surfactant phase, extracting solvent, thiazolylazo dyes

When aqueous micellar solutions of nonionic surfactants having an appropriate oxyethylene moiety are heated, they become turbid within a narrow temperature range of the incipient phase separation, called the cloud point. At a higher temperature than the cloud point the solutions separate into two distinct phases: ${ }^{1}$ an almost micelle-free aqueous solution (aqueous phase) of the surfactants at a concentration below or equal to their critical micelle concentration, and a surfactantrich phase (surfactant phase).

This phase separation can be used for the extraction of metal chelates. ${ }^{2,3}$ When poly(oxyethylene) $=4$-nonylphenyl =ether with oxyethylene units 7.5 (PONPE7.5) is used, the extraction can be made at room temperature without heating the micellar solutions, since the cloud point for a dilute solution of PONPE7.5 is located far below room temperature. The surfactant phase, thus obtained, consists of $17.8 \%$ $(\mathrm{w} / \mathrm{w})$ PONPE-7.5 and $82.2 \%(\mathrm{w} / \mathrm{w})$ water at $293 \mathrm{~K}, 4$ and is an isotropic solution when observed by a polarizing microscope.

We have already reported the distribution equilibria of some chelating reagents and their metal chelates between the two phases. ${ }^{5-7}$ The distribution equilibria were found to be solved in the same manner as described regarding extraction studies with ordinary organic solvents. In the present study, the partition constants of four thiazolylazophenols and their metal chelates were investigated in order to clarify the influence of the molecular size on the partition constants and, hence, to elucidate the properties of PONPE-7.5 as an extracting solvent. Furthermore, we compared the partition constants with those obtained between water and octanol, since the surfactant phase of PONPE-7.5 is expected to have properties similar to those of alcohol- and ether-type organic solvents.

\section{Experimental}

\section{Instruments}

The amounts of metal chelates extracted into the surfactant phase were measured with a double-beam spectrophotometer (Hitachi model 320) equipped with $1-\mathrm{cm}$ cells and with an atomic absorption spectrophotometer (Hitachi model 170-30). A pH meter (Toa model HM-15A) was used for $\mathrm{pH}$ measurements.

\section{Reagents}

Standard solutions of iron(II), nickel(II), zinc(II) and cadmium(II) were prepared by first dissolving their nitrate salts in $0.01 \mathrm{~mol} \mathrm{dm}^{-3}$ perchloric acid and then standardizing by EDTA titration. 2-(2-Thiazolylazo)-4-methylphenol (TAC, a) was prepared from thiazolyldiazonium chloride and 4-methylphenol. ${ }^{8}$ Other chelating reagents, 2-(2-thiazolylazo)-4-methoxyphenol (TAMP, b), 2-(2-thiazolylazo)-4-ethoxyphenol (TAEP, c), and 2-(2-thiazolylazo)-4-phenylphenol 


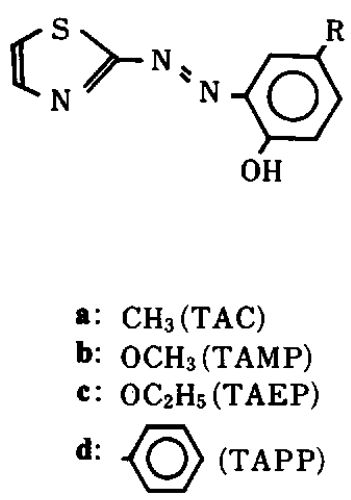

(TAPP, d), were obtained by a similar method. The purities and structures of these chelating reagents were verified by HPLC (detected at $254 \mathrm{~nm}$ ), H-NMR and IR. Each chelating reagent solution was prepared by dissolving it in a $0.1 \mathrm{~mol} \mathrm{dm}^{-3}$ sodium hydroxide solution. An aqueous $20.0 \%(\mathrm{w} / \mathrm{w})$ solution of PONPE7.5 (cloud point: $299 \mathrm{~K},{ }^{3}$ Tokyo Kasei Industries) and a PONPE-20 solution (average units of oxyethylene, 20; cloud point, $373 \mathrm{~K} ;{ }^{3}$ Tokyo Kasei Industries) were prepared by dissolving $20.0 \mathrm{~g}$ of each in $80.0 \mathrm{~g}$ of water. Buffer solutions were prepared by mixing $0.1 \mathrm{~mol} \mathrm{dm}^{-3}$ perchloric acid or a sodium hydroxide solution with a $0.1 \mathrm{~mol} \mathrm{dm}^{-3}$ aqueous solution of the following specified buffer component: sodium acetate $(\mathrm{pH}=1-5.5)$, 2-( $N$-morpholino)ethanesulfonic acid ( $\mathrm{pH}=5.5-6.5)$, 3 - $(N$-morpholino)propanesulfonic acid $(\mathrm{pH}=6.5-7.5)$, tris(hydroxymethyl)aminomethane $(\mathrm{pH}=7.5-9)$ and 3-cyclohexylaminopropanesulfonic acid $(\mathrm{pH}=9-12)$.

Other reagents used were guaranteed grade and their solutions were prepared by the same manner as reported previously. ${ }^{4-7}$

\section{Procedure}

Extraction with PONPE-7.5. Prescribed amounts of a PONPE-7.5 $(0.250 \mathrm{~g}$ of $20.0 \%(\mathrm{w} / \mathrm{w})$ solution), a chelating reagent, a metal ion (if necessary), sodium perchlorate and a buffer component were placed into a centrifuge tube. The mixture was made up to $50.00 \mathrm{~g}$ with water (initial solution). The cloud point of the initial solution locates at $274 \mathrm{~K}^{3}$ The total concentration of the buffer component was maintained below $5 \times 10^{-3} \mathrm{~mol} \mathrm{dm}^{-3}$, and the ionic strength was kept constant at 0.1 with sodium perchlorate. The turbid solution, thus prepared, was kept at $293 \mathrm{~K}$ in a water bath for $30 \mathrm{~min}$ for equilibration and then spun in a centrifuge at $10000 \mathrm{~min}^{-1}$ for $1 \mathrm{~min}$. Then, most of the aqueous phase was removed. Upon cooling for $50 \mathrm{~min}$ (about $253 \mathrm{~K}$ ), the surfactant phase became viscous and, thus, the aqueous phase which remained in the tube could simply be poured off. The $\mathrm{pH}$ value of the aqueous phase was measured at $293 \mathrm{~K}$. A $1-\mathrm{cm}^{3}$ portion of the $20.0 \%(w / w)$ PONPE-20 solution was added to the surfactant phase in order to increase the cloud point of the solution. For metal ion extraction, a $2 \mathrm{~cm}^{3}$-aliquot of the chelating reagent solution and
Table 1 Spectral data for thiazolylazo dyes and their chelates under optimum pH condition

\begin{tabular}{lccr}
\hline Compound & $\lambda_{\max } / \mathrm{nm}$ & $\begin{array}{c}\varepsilon / \\
10^{4} \mathrm{dm}^{3} \mathrm{~mol}^{-1} \mathrm{~cm}^{-1}\end{array}$ & $\mathrm{pH}$ \\
\hline TAC & 373 & 0.982 & 7.0 \\
TAMP & 560 & 1.52 & 12.5 \\
TAEP & 558 & 1.46 & 12.5 \\
TAPP & 550 & 1.60 & 12.5 \\
{$\left[\mathrm{Fe}(\mathrm{tac})_{2}\right]$} & 751 & 0.935 & 5.5 \\
{$\left[\mathrm{Ni}(\mathrm{tac})_{2}\right]$} & 610 & 2.43 & 7.0 \\
{$\left[\mathrm{Fe}(\mathrm{tamp})_{2}\right]$} & 778 & 1.39 & 5.5 \\
{$\left[\mathrm{Ni}(\mathrm{tamp})_{2}\right]$} & 620 & 3.24 & 6.8 \\
{$\left[\mathrm{Fe}(\operatorname{taep})_{2}\right]$} & 780 & 1.35 & 5.5 \\
{$\left[\mathrm{Ni}(\mathrm{taep})_{2}\right]$} & 625 & 3.33 & 6.8 \\
{$[\mathrm{Fe}($ tapp } & \\
{$\left[\mathrm{Ni}(\mathrm{tapp})_{2}\right]$} & 764 & 1.43 & 5.5 \\
\hline
\end{tabular}

$2 \mathrm{~cm}^{3}$ of an appropriate buffer solution were also added to the surfactant phase. The resulting clear solution was diluted to $10.0 \mathrm{~g}$ with water (final solution; cloud point, $323 \mathrm{~K}$ ). ${ }^{3}$ The absorbance of the final solution was measured against a reagent blank for nickel(II) and iron(II) chelates under the conditions shown in Table 1. Cadmium(II) and zinc(II) chelates in the final solution were determined by atomic absorption spectrometry (AAS). Calibration curves of cadmium(II) and zinc(II) were prepared according to the procedure from which dilution to $50.00 \mathrm{~g}$ with water, centrifuging, and addition of sodium perchlorate were omitted.

The concentration of a metal ion extracted into the surfactant phase was calculated on the basis of a material balance of the metal ion and the volume of the surfactant phase $\left(v_{\mathrm{s}}: 0.302 \mathrm{~cm}^{3}\right.$ at $\left.293 \mathrm{~K}\right)$. The distribution ratio $(q)$ was defined as the ratio of the total concentration of the metal ion in the surfactant phase to that in the aqueous phase. The latter was calculated from the material balance and the volume of the aqueous phase $\left(v_{\mathrm{w}}: 49.11 \mathrm{~cm}^{3}\right.$ at $\left.293 \mathrm{~K}\right)$.

The volume of the surfactant phase $\left(v_{s}\right)$ under the experimental conditions was calculated using the following relation: ${ }^{4}$

$$
v_{\mathrm{s}}=R / d_{\mathrm{s}} W,
$$

where $d_{s}$ is the density of the surfactant phase $(1.016 \mathrm{~g}$ $\left.\mathrm{cm}^{-3}\right), W$ the PONPE-7.5 concentration $(0.178 \mathrm{~g} / \mathrm{g})$ and $R$ the weight of the PONPE-7.5 $(0.1092 \mathrm{~g})$. The volume of the aqueous phase was obtained by the use of the density of the aqueous phase $\left(1.012 \mathrm{~g} \mathrm{~cm}^{-3}\right)$.

Extraction with octanol. A $20-\mathrm{cm}^{3}$ aliquot of an aqueous solution containing a metal ion (if necessary), sodium perchlorate $\left(0.1 \mathrm{~mol} \mathrm{dm}^{-3}\right)$ and a buffer component were placed in a $50-\mathrm{cm}^{3}$ centrifuge tube. An octanol solution $\left(5 \mathrm{~cm}^{3}\right)$ containing TAC or TAMP was also added to the tube; the tube was shaken for $1 \mathrm{~h}$ at room temperature $(293 \mathrm{~K})$. After phase separation, the $\mathrm{pH}$ value of the aqueous phase was measured. The 
amount of iron(II) and nickel(II) in the octanol phase were determined spectrophotometrically. Cadmium(II) and zinc(II) in the aqueous phase were determined by AAS.

\section{Results and Discussion}

\section{Distribution equilibria of chelating reagents}

A plot of the logarithmic distribution ratio for TAC as a function of $\mathrm{pH}$ is shown in Fig. 1. The distribution ratio $(q)$ is constant in the $\mathrm{pH}$ region between 2 and 5.5, where the value of $q$ is equal to the partition constant $\left(K_{\mathrm{d} 1}\right)$ of the uncharged species (HL). This fact also means that the contribution of a protonated form $\left(\mathrm{H}_{2} \mathrm{~L}^{+}\right)$can be neglected. Above a $\mathrm{pH}$ of 10.5 , the value of $q$ apparently tends to be constant, suggesting an extraction of the TAC anion $\left(\mathrm{L}^{-}\right)$. Thus, the distribution ratio can be described by

$$
\begin{aligned}
q & =\frac{[\mathrm{HL}]_{\mathrm{s}}+\left[\mathrm{L}^{-}\right]_{\mathrm{s}}}{[\mathrm{HL}]+\left[\mathrm{L}^{-}\right]} \\
& =\frac{K_{\mathrm{d} 1}+K_{\mathrm{d} 2}^{\prime} K_{\mathrm{a}}\left[\mathrm{H}^{+}\right]^{-1}}{1+K_{\mathrm{a}}\left[\mathrm{H}^{+}\right]^{-1}},
\end{aligned}
$$

where subscript $\mathrm{s}$ is the surfactant phase, $K_{\mathrm{a}}$ the acid dissociation constant of $\mathrm{HL}$, and $K_{\mathrm{d} 1}$, and $K_{\mathrm{d} 2}^{\mathrm{h}}$ the partition constants of $\mathrm{HL}$ and $\mathrm{L}^{-}$, respectively, as defined by

$$
\begin{aligned}
& K_{\mathrm{a}}=\left[\mathrm{H}^{+}\right]\left[\mathrm{L}^{-}\right] /[\mathrm{HL}], \\
& K_{\mathrm{d} 1}=[\mathrm{HL}]_{\mathrm{s}} /[\mathrm{HL}],
\end{aligned}
$$

and

$$
K_{\mathrm{d} 2}^{\prime}=\left[\mathrm{L}^{-}\right]_{\mathrm{s}} /\left[\mathrm{L}^{-}\right]
$$

The ion $\mathrm{L}^{-}$may be extracted as ionic associates of the type $\mathrm{Na}^{+} \mathrm{L}^{-}$. For brevity, the extraction equilibrium of the ionic associate was described with a conditional constant $\left(K_{\mathrm{d} 2}^{\prime}\right)$ in which the concentration term of the counter ion was omitted.

The acid dissociation constant $\left(K_{\mathrm{a}}\right)$ and the partition constants $\left(K_{\mathrm{d} 1}\right.$ and $\left.K_{\mathrm{d} 2}^{\prime}\right)$ of TAC were calculated through a least-square fit of the model described by Eq. (1) to the data. These constants are listed in Tables 2 and 3. The solid line for TAC in Fig. 1 calculated from these constants shows good agreement with the experimental points. The equilibrium constants of other chelating reagents are also summarized in Tables 2 and 3.

\section{Distribution equilibria of metal chelates}

Figure 1 shows the distribution curves of four metal chelates with TAC as a function of $\mathrm{pH}$. The values of $q$ tend to be constant above a certain $\mathrm{pH}$. This is ascribable to the preferential extraction of an uncharged chelate $\left(\mathrm{ML}_{2}\right)$. The distribution ratio of a

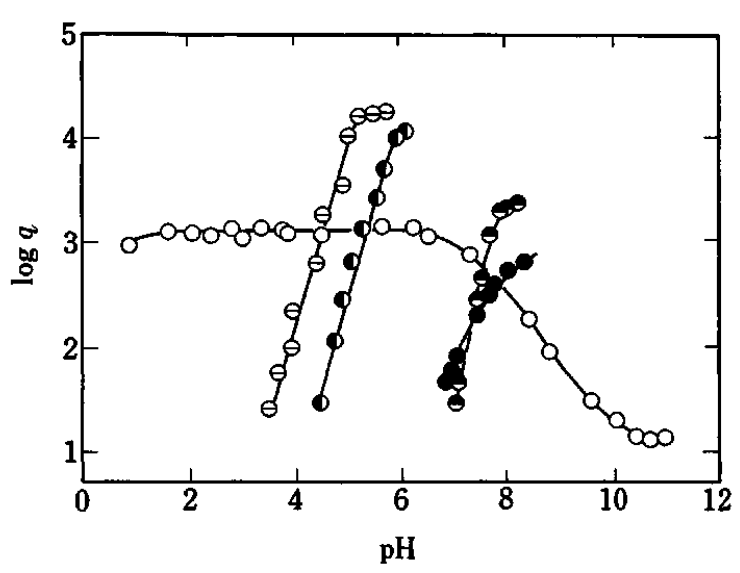

Fig. 1 Distribution curves for TAC and its metal chelates at $293 \mathrm{~K}$ and ionic strength, $0.10\left(\mathrm{NaClO}_{4}\right)$. (O) TAC, $[\mathrm{TAC}]=9.81 \times 10^{-6} \mathrm{~mol} \mathrm{dm}^{-3} ;(\Theta) \mathrm{Fe}(\mathrm{II}),\left[\mathrm{Fe}^{2+}\right]=4.00 \times 10^{-6}$ $\mathrm{mol} \mathrm{d} \mathrm{m}{ }^{-3}$, [TAC] $=8.24 \times 10^{-5} \mathrm{~mol} \mathrm{~d} \mathrm{~m}^{-3}$; (D) $\mathrm{Ni}(\mathrm{II})$, $\left[\mathrm{Ni}^{2+}\right]=4.04 \times 10^{-6} \mathrm{~mol} \mathrm{dm}^{-3},[\mathrm{TAC}]=8.24 \times 10^{-5} \mathrm{~mol} \mathrm{dm}^{-3} ;(\mathrm{O})$ $\mathrm{Zn}(\mathrm{II}), \quad\left[\mathrm{Zn}^{2+}\right]=3.94 \times 10^{-6} \mathrm{~mol} \mathrm{dm}^{-3}, \quad[\mathrm{TAC}]=8.24 \times 10^{-5}$ $\mathrm{mol} \mathrm{dm}{ }^{-3}$; (Ө) $\mathrm{Cd}(\mathrm{II}),\left[\mathrm{Cd}^{2+}\right]=4.00 \times 10^{-6} \mathrm{~mol} \mathrm{~d} \mathrm{~m}^{-3}$, $[\mathrm{TAC}]=8.24 \times 10^{-5} \mathrm{~mol} \mathrm{dm}^{-3}$.

divalent metal ion $\left(\mathrm{M}^{2+}\right)$ with TAC can be found using Eq. (2). The distribution of a charged chelate $\left(\mathrm{ML}^{+}\right)$ was found to be negligible. Equation (2):

$$
q=\frac{\left[\mathrm{ML}_{2}\right]_{s}}{\left[\mathrm{M}^{2+}\right]+\left[\mathrm{ML}^{+}\right]+\left[\mathrm{ML}_{2}\right]},
$$

can be rewritten in terms of the equilibrium concentration for $\mathrm{L}^{-}$in the aqueous phase:

$$
q=\frac{K_{\mathrm{D}} \beta_{2}\left[\mathrm{~L}^{-}\right]^{2}}{1+\beta_{1}\left[\mathrm{~L}^{-}\right]+\beta_{2}\left[\mathrm{~L}^{-}\right]^{2}},
$$

where $K_{\mathrm{D}}$ is the partition constant of $\mathrm{ML}_{2}$, and $\beta_{1}$ and $\beta_{2}$ are the overall stability constants. These are defined as

$$
\begin{aligned}
& K_{\mathrm{D}}=\left[\mathrm{ML}_{2}\right]_{\mathrm{s}} /\left[\mathrm{ML}_{2}\right], \\
& \beta_{1}=\left[\mathrm{ML}^{+}\right] /\left[\mathrm{M}^{2+}\right]\left[\mathrm{L}^{-}\right],
\end{aligned}
$$

and

$$
\beta_{2}=\left[\mathrm{ML}_{2}\right] /\left[\mathrm{M}^{2+}\right]\left[\mathrm{L}^{-}\right]^{2} .
$$

The concentration of $\mathrm{L}^{-}$in the aqueous phase was computed according to the following equation, derived from the material balance of TAC:

$$
[\mathrm{L}]_{\mathrm{t}}=\frac{v_{\mathrm{s}} \Sigma[\mathrm{L}]_{\mathrm{s}}+v_{\mathrm{w}} \Sigma[\mathrm{L}]}{v_{\mathrm{s}}+v_{\mathrm{w}}},
$$

Here, $[\mathrm{L}]_{\mathrm{t}}$ is the total concentration of TAC, taken to be entirely in the initial solution $\left(v_{\mathrm{s}}+v_{\mathrm{w}}\right)$, and $\Sigma[\mathrm{L}]_{\mathrm{s}}$ and $\Sigma[L]$ are the total concentrations of TAC in the surfactant and in the aqueous phases, respectively. The amounts of TAC bound to a metal ion were assumed to 


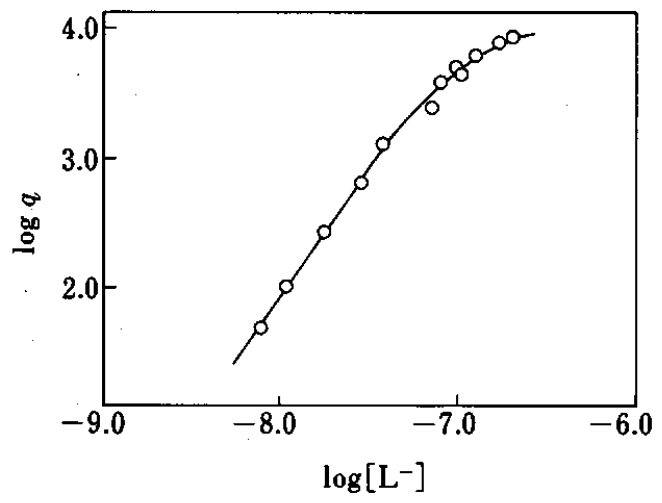

Fig. 2 Plot of $\log q$ vs. $\log \left[\mathrm{L}^{-}\right]$for iron(II) with TAC.

be negligible, since the metal ion was extracted by adding about 20 -fold excess of TAC in relation to the metal concentrations.

A plot of $\log q$ vs. $-\log \left[\mathrm{L}^{-}\right]$for the iron(II) chelate with TAC is shown in Fig. 2. The partition and the stability constants for the iron(II) chelate were calculated through a least-square fit of the model described by Eq. (3) to the plot in Fig. 2. The solid line in Fig. 2, calculated with the constants for the iron(II) chelate shown in Tables 2 and 3, fits very well to the experimental plots. These constants of other metal chelates were also determined in the same manner as described above, and are summarized in Tables 2 and 3 .

The chelating reagents used are tridentate ligands; thus, they may react with these metal ions to form coordination-saturated $\mathrm{ML}_{2}$ type chelates. In the conventional solvent extraction of the chelate with a nonpolar solvent the following relation is expected from the regular solution theory:9

$$
\log K_{\mathrm{D}} \simeq 2 \log K_{\mathrm{dl}} .
$$

As can be seen in Table 3, all the $\log K_{\mathrm{D}}$ values of the chelates are considerably smaller than $2 \log K_{d 1}$ for the respective chelating reagents. This is often encountered in extraction with polar organic solvents..$^{10}$

The regular solution theory also predicts that the partition constants $\left(K_{\mathrm{D}}\right)$ will be almost independent of metal ions. However, the constants in Table 3 vary with the kind of metal ions. This is especially apparent in chelates with TAC; the logarithmic $K_{\mathrm{D}}$ value of the iron(II) chelate is one order of magnitude larger than that of the zinc(II) chelate. In chelates with TAMP and TAEP the difference in the $\log K_{\mathrm{D}}$ values is small. These facts suggest that the extraction mechanism in the present system is different from that in ordinary extraction.

For a further elucidation of the properties of PONPE-7.5, we examined the distribution equilibria of three chelating reagents and three TAC chelates between water and octanol phases. The distribution equilibria were determined in the same way as in the
Table 2 Acid dissociation constants $\left(\mathrm{p} K_{\mathrm{a}}\right)$ of thiazolylazodyes and the stability constants $\left(\log \beta_{2}\right)$ of their metal chelates

\begin{tabular}{llllll}
\hline & \multirow{2}{*}{$\mathrm{p} K_{\mathrm{a}}$} & \multicolumn{4}{c}{$\log \beta_{2}$} \\
\cline { 3 - 6 } & & $\mathrm{Fe}(\mathrm{II})$ & $\mathrm{Ni}(\mathrm{II})$ & $\mathrm{Cd}(\mathrm{II})$ & $\mathrm{Zn}(\mathrm{II})$ \\
\hline TAC & 8.07 & 15.50 & 14.00 & 9.84 & 10.60 \\
TAMP & 7.86 & 16.50 & 15.61 & 10.24 & 11.00 \\
TAEP & 7.86 & 15.44 & 15.04 & 13.04 & 10.80 \\
TAPP & 8.21 & 18.88 & 15.90 & 12.44 & 12.42 \\
\hline
\end{tabular}

lonic strength=0.1 ( $\left.\mathrm{NaClO}_{4}\right), 293 \mathrm{~K}$.

Table 3 Partition constants of thiazolylazo dyes and their metal chelates

\begin{tabular}{lcccccc}
\hline & & & \multicolumn{5}{c}{$\log K_{\mathrm{D}}$} \\
\cline { 4 - 7 } & $\log K_{\mathrm{d} 1}$ & $\log K_{\mathrm{d} 2}^{\prime}$ & $\mathrm{Fe}(\mathrm{II})$ & $\mathrm{Ni}(\mathrm{II})$ & $\mathrm{Cd}(\mathrm{II})$ & $\mathrm{Zn}(\mathrm{II})$ \\
\hline TAC & 3.11 & 1.14 & 4.23 & 4.03 & 3.29 & 3.08 \\
TAMP & 2.24 & 0.95 & 2.48 & 2.71 & 3.14 & 2.95 \\
TAEP & 2.50 & 0.09 & 3.07 & 3.21 & 3.25 & 3.06 \\
TAPP & 3.63 & 2.05 & 2.80 & 3.77 & 2.71 & 3.19 \\
\hline
\end{tabular}

Ionic strength $=0.10\left(\mathrm{NaClO}_{4}\right), 293 \mathrm{~K}$.

case of the extraction with PONPE-7.5.

\section{Comparison with octanol extraction system}

The partition constants of three chelating reagents (TAC, TAMP and 8-hydroxyquinoline ${ }^{4}$ ) in the present system were compared with those in the octanol system. The partition constant of 8-hydroxyquinoline between water and octanol was given by Seiler. ${ }^{11}$ As Fig. 3 shows, there is a good linear relationship between the partition constants in the present system and those in the octanol system. Such a linear free-energy relationship is observed in conventional solvent extraction, when two organic solvents used have the same properties. ${ }^{10}$ Therefore, the extraction of the chelating reagents with PONPE-7.5 is ascribable to a specific interaction, such as hydrogen bonding, between functional groups on the chelating reagents and ether oxygen (or $\mathrm{OH}$ group) on the oxyethylene chain of PONPE7.5.

On the other hand, no relationships exist regarding in the extraction of the chelates with TAC, as shown in Fig. 3. This fact clearly shows that the distribution mechanism of the chelates is different from that of the chelating reagents. An interaction causes the chelates to remain in a hydrophobic part, the solvent structure of which is different from that of the octanol phase.

\section{Properties of PONPE-7.5}

One of the features of PONPE-7.5 as an extracting solvent is that the partition constants of the chelates vary with the kind of metal ions. In Fig. 3, the partition constants depend on the kind of metal ions, 


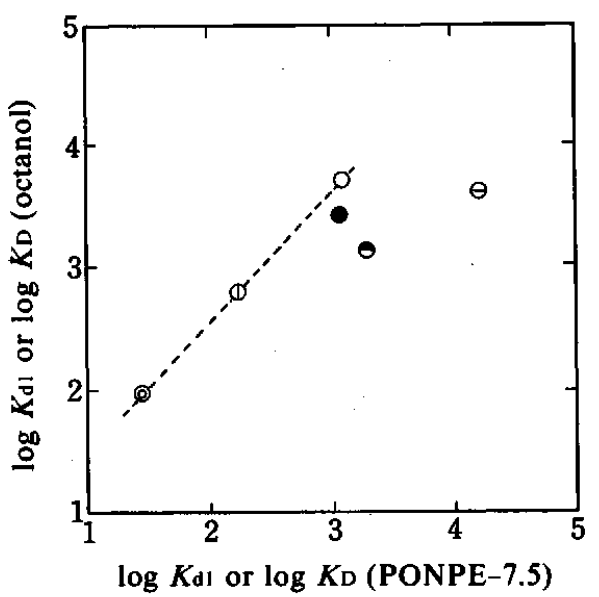

Fig. 3 Correlation between the partition constants in an aqueous-octanol system and those in a PONPE-7.5 system. (@) 8-hydroxyquinoline; (D) TAMP; (O) TAC; (O) [Zn(tac) $\left.)_{2}\right] ;(\Theta)\left[\mathrm{Cd}(\operatorname{tac})_{2}\right] ;(\Theta)\left[\mathrm{Fe}(\operatorname{tac})_{2}\right]$.

whereas the partition constants in an octanol system are almost independent of metal ions. These results could be ascribable to the presence of microscopically ordered structures in the surfactant phase, such as a liquid crystal ${ }^{12-14}$, which can differentiate slight differences in the molecular size, shape and structural factors.

However, when observed by a polarizing microscope in the present study, the surfactant phase $(17.8 \%(w / w)$ PONPE-7.5) was isotropic, while a certain liquid crystalline was confirmed to be present in $80 \%(w / w)$ PONPE-7.5. The phase transition from the isotropic phase to the liquid crystalline phase is not continuous; thus, some ordered structures are not likely to be present in the surfactant phase.

On the other hand, Shinohara et al..$^{15}$ have reported the phase transition of a thermotropic liquid crystal by using the ESR technique with a spin probe incorporated in a nematic liquid crystal. They demonstrated the existence of the short-range order of the nematic phase: in other words, the presence of some microscopically ordered structure, even in the range of the isotropic phase.

From these considerations, the surfactant phase must comprise two hydrophobic strucures: one is an isotropic structure and the other is a microscopically ordered structure, such as liquid crystals. Therefore, the variation of the partition constants for the TAC and TAPP chelates is due to their incorporation into the microscopically ordered structure. On the other hand, the partition constants of the TAMP and TAEP chelates, being almost independent of metal ions, may be mainly explained in terms of a hydrophobic inter- action with the nonylphenyl group on PONPE-7.5 in the isotropic structure. As already pointed out, the chelating reagents are distributed to the hydrophilic part, probably in the isotropic structure. All these factors would be responsible for the partition of the chelating reagents and their metal chelates. Regarding these respects, further studies must be carried out.

Finally, it is important to point out that the present technique has been extended to the separation of membrane proteins. ${ }^{16,17}$ This can not be achieved with ordinary solvent extraction, since organic solvents strongly denature proteins. The results obtained in the present study also suggest the possibilities of the separation of proteins and other biochemical species on the basis of differences in the molecular size, shape and various structural factors.

\section{References}

1. K. Shinoda, T. Nakagawa, B. Tamamushi and T. Isemura, "Colloidal Surfactants", p. 129, Academic Press, New York, London, 1963.

2. H. Watanabe, in "Solution Behavior of Surfactants", Vol. 2, p. 1035, ed. K. L. Mittal and E. J. Fendler, Plenum Press, New York, 1982.

3. H. Watanabe and H. Tanaka, Talanta, 25, 585 (1978).

4. H. Hoshino, T. Saitoh, H. Taketomi, T. Yotsuyanagi, H. Watanabe and K. Tachikawa, Anal. Chim. Acta, 147, 339 (1983).

5. S. Kawamorita, H. Watanabe and K. Haraguchi, Anal. Sci., 1, 41 (1985).

6. S. Kawamorita, H. Watanabe, K. Haraguchi and M. Miyajima, Nippon Kagaku Kaishi, 1986, 901.

7. H. Watanabe, T. Kamidate, S. Kawamorita, K. Haraguchi and M. Miyajima, Anal. Sci., 3, 433 (1987).

8. G. Nakagawa and H. Wada, Nippon Kagaku Kaishi, 85, 54 (1964).

9. N. Suzuki, K. Akiba, Bull. Chem. Soc. Jpn., 41, 1854 (1968).

10. A. Leo, C. Hansch and D. Elkins, Chem. Rev., 71, 523 (1971).

11. J. Seiler, Eur. J. Med. Chem., 9, 473 (1974).

12. H. Tsutsumi, Nippon Kagaku Kaishi, 1981, 1691.

13. K. Larsson, Z. Phys. Chem., 56, 173 (1967).

14. N. Krog and K. Larsson, Chem. Phys. Lipids, 2, 129 (1968).

15. Y. Shinohara, M. Shiotani and J. Sohma, Jpn. J. Appl. Phys., 16, 1437 (1977).

16. C. Bordier, J. Boil. Chem., 256, 1604, (1981).

17. T. Bouvier, R. J. Etges and C. Bordier, J. Boil. Chem., 260, 15504 (1985).

(Received November 21, 1988)

(Accepted July 6, 1989) 